\title{
All-organic Solution Processed Solar Cells Based on Terephthaladehyde Self-assembled Monolayers
}

\author{
Chetan Jagdish Bhongale* and Rahul Chaudhari
}

\begin{abstract}
An acetalization reaction was used as a versatile method to immobilize terephthaldehyde molecules on the Indium tin oxide (ITO) surface to fabricate the Poly(3-hexylthiophene-2,5-diyl) ( $\left.\mathrm{P}_{3} \mathrm{HT}\right)-(6,6)$-Phenyl-C61-butyric acid methyl ester (PCBM) based all-organic solution processed solar cells and their performance was compared with the devices with poly(3,4ethylenedioxythiophene) polystyrene sulfonate (PEDOT:PSS) as a buffer layer.
\end{abstract}

Keywords: Organic solar cells; $\mathrm{P}_{3}$ HT-PCBM; Self-assembled monolayers (SAM); Photovoltaics; Terephthaldehyde.

Received: 18 January 2021; Accepted date: 20 April 2021.

Article type: Research article.

\section{Introduction}

In recent years, photovoltaic (PV) devices based on polymerfullerene thin films have been widely investigated because of solution processing, possible capability and flexibility of large area device. ${ }^{[1-3]}$ During the last ten years, research efforts have focused on poly(alkyl-thiophenes), and in particular on $\mathrm{P}_{3} \mathrm{HT}$ which has outcast poly[2-methoxy-5-(3,7-dimethyloctyloxy)1,4-phenylenevinylene] (MDMO-PPV), MDMO-PPV like polymers because of their large gap and low mobility. With the report of the observation of short-circuit current density of $8.7 \mathrm{~mA} \mathrm{~cm}^{-2}{ }^{[4]}$ in an organic solar cell, there appears to be rapid development for the Poly(3-hexylthiophene-2,5diyl)-(6,6)-Phenyl-C61-butyric acid methyl ester $\left(\mathrm{P}_{3} \mathrm{HT}: \mathrm{PCBM}\right)$ blend, followed by first explicit reports on efficiency enhancement in $\mathrm{P}_{3} \mathrm{HT}$ :PCBM cells as a result of thermal annealing. ${ }^{[5]}$ The field of bulk heterojunction $(\mathrm{BHJ})$ is mainly dominated by $\mathrm{P}_{3} \mathrm{HT}: \mathrm{PCBM}$ system, with reproducible efficiencies approaching 5\% ${ }^{[2,6,7]}$ and improvement for the device performance have been made by new designs and materials $^{[8]}$ and synthesis of low band-gap polymers, morphology tuning of the bulk heterojunction, newer donoracceptor combinations etc. ${ }^{[9]}$ In addition to these, the improvement of interface between the organic materials and the electrodes is critical to device performance. ${ }^{[10]}$ Understanding and tuning the charge injection barrier between electroactive conjugated molecule overlayers and metal or

Defence Institute of Advanced Technology (DU), Pune-411025, India.

*E-mail: chetanjb@diat.ac.in (C. Bhongale) semiconductor substrates is important. Indium tin oxide (ITO) is widely used as the anode in organic PV cells, because of its transparency, low resistivity, ease of patterning, and wide availability at low cost. ${ }^{[11]}$ Despite these favorable qualities, the hydrophilic nature of the ITO surface makes it incompatible with organic materials and also the relatively low work function of ITO results in contact resistance in the device through a reduced rate of hole transfer between ITO and the organic film.

To improve the properties of ITO electrode, various treatments such as ozone treatment, ${ }^{[12]}$ oxygen-plasma etching, ${ }^{[13]}$ UV - spin-coating a thin layer of poly $(3,4$ ethylenedioxythiophene)/poly(styrene sulfonic acid) (PEDOT:PSS), ${ }^{[14]}$ and self-assembling a monolayer of dipolar molecules ${ }^{[11,15]}$ onto the ITO substrates have been performed. This leads to an increase in the ITO work function due to changes in surface morphology and chemical structure. In this work we used UV-ozone treatment followed by surface modification by self-assembled monolayer (SAM) of terephthaldehyde (TPA) which has functional aldehyde groups. Although poly (3,4-ethylenedioxythiophene) polystyrene sulfonate (PEDOT:PSS) is widely used to coat the ITO, it may have a chemical reaction with anode as oxygen treated ITO is highly reactive. ${ }^{[16]}$ However, ITO surface can be stabilized by modification with SAM. The commonly accepted energy conversion process is comprised of four fundamental steps: absorption of light and generation of excitons, diffusion of excitons, dissociation of the excitons with generation of charge, and charge transport and charge 
(a)



(b)
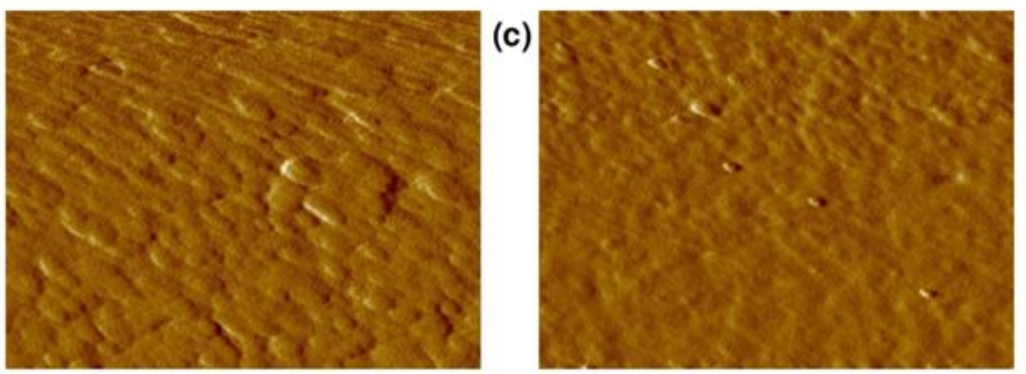

Fig. 1 (a) Schematic illustration of TMS silylation and TPA acetalization on the ITO substrate and AFM images of (b) bare ITO substrate (c) terephthaladehyde molecules immobilzed on ITO surface. The area of each image is $2 \times 2 \mu \mathrm{m}^{2}$.

collection. It is apparent that the active layer donor-acceptor composite governs all aspects of this energy process, with the exception of charge collection, wich is based on the electronic interface between the active layer composite and the respective electrode. ${ }^{[1]}$ In this Communication, we report the fabrication and stability studies of all-organic solution processed solar cells based on self-assembled monolayer of terephthaldehyde molecules immobilized on ITO by acetalization reaction and their comparative study with the device with PEDOT:PSS as buffer layer.

\section{Experimental details}

Terephthaladehyde (TPA) SAM was formed in two steps. First, a trimethylsilyl (TMS) terminated surface was formed using a sylilation reaction between a chloride groups of trimethylsilylchloride (TMSC) and hydroxyl groups of the pre-treated ITO surface (see Fig. 1a). And then terephthaladehyde was immobilized on the surfaces using an acetalization reaction between TMS-terminated surface and and aldehyde group of TPA. Our previous studies of contact angle, X-ray photoelectron and UV-absorption spectra on TPA molecules revealed that TPA molecules on these surfaces are well-packed with a high surface density. ${ }^{[17]}$ The bare glass substrates (substrate size: $25 \times 25 \times 1 \mathrm{~mm}$ ) coated with a 100 $\mathrm{nm}$ ITO layer were cleaned using ultrasonication in acetone, detergent, pure water and isopropanol to remove residual organic materials. The UV-Ozone treatment on ITO surface was done for $30 \mathrm{~min}$ to form hydroxyl groups. A $10 \mathrm{mM}$ solution (dehydrated toluene) and a $10 \mathrm{mM}$ TPA solution (dehydrated dichloromethane) were prepared in Teflon containers. The cleaned substrates were soaked in the TMSC solution for $1 \mathrm{~h}$ to terminate the substrate surfaces with TMS using a silylation reaction. The TMS-terminated substrates, after rising, were soaked in the TPA solution for $18 \mathrm{~h}$ to immobilize the TPA on the substrate surfaces using an acetalization reaction. Then these substrates were cleaned by ultrasonication in pure dichloromethane to remove physisorbed molecules from the surfaces. Fig. 1-b shows the Atomic Force Microscopic (AFM) image of the bare ITO substrate having grain-like morphology with surface roughness of about $0.9 \mathrm{~nm}$ and after the immobilization of TPA molecules on its surface, the surface roughness was still $0.8 \mathrm{~nm}$ (Fig.1-c) indicating that monolayer does not affect the morphology of ITO. The AFM images were obtained with Keyence Nanoscale Hybrid Microscope VN-8010. The silylation and acetalization reactions were performed in a nitrogen-filled glove box at room temperature. These substrates were dried in nitrogen filled glove box prior depositing active layers and device fabrication.

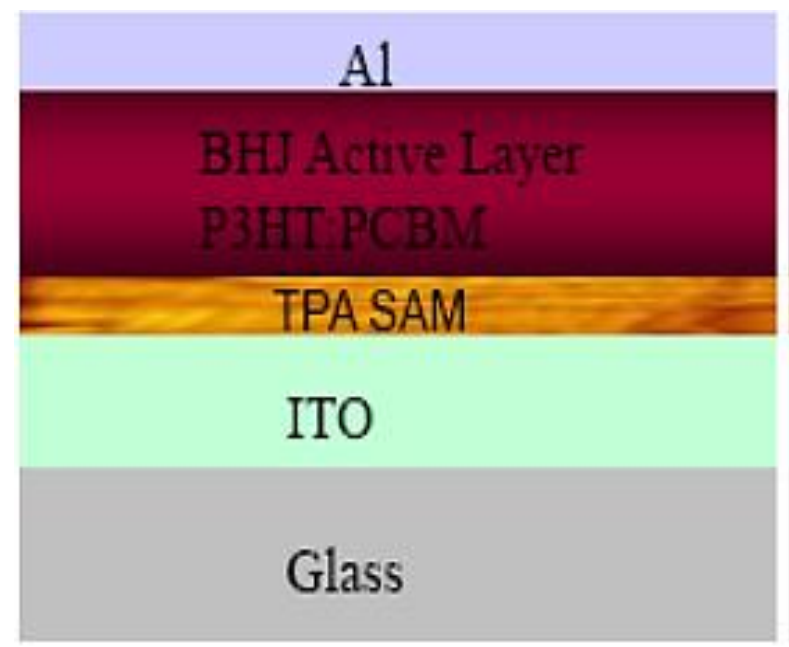

Fig. 2 Device architecture of the TPA-SAM modified $\mathrm{P}_{3} \mathrm{HT}$ :PCBM based bulk heterojunction solar cells. 

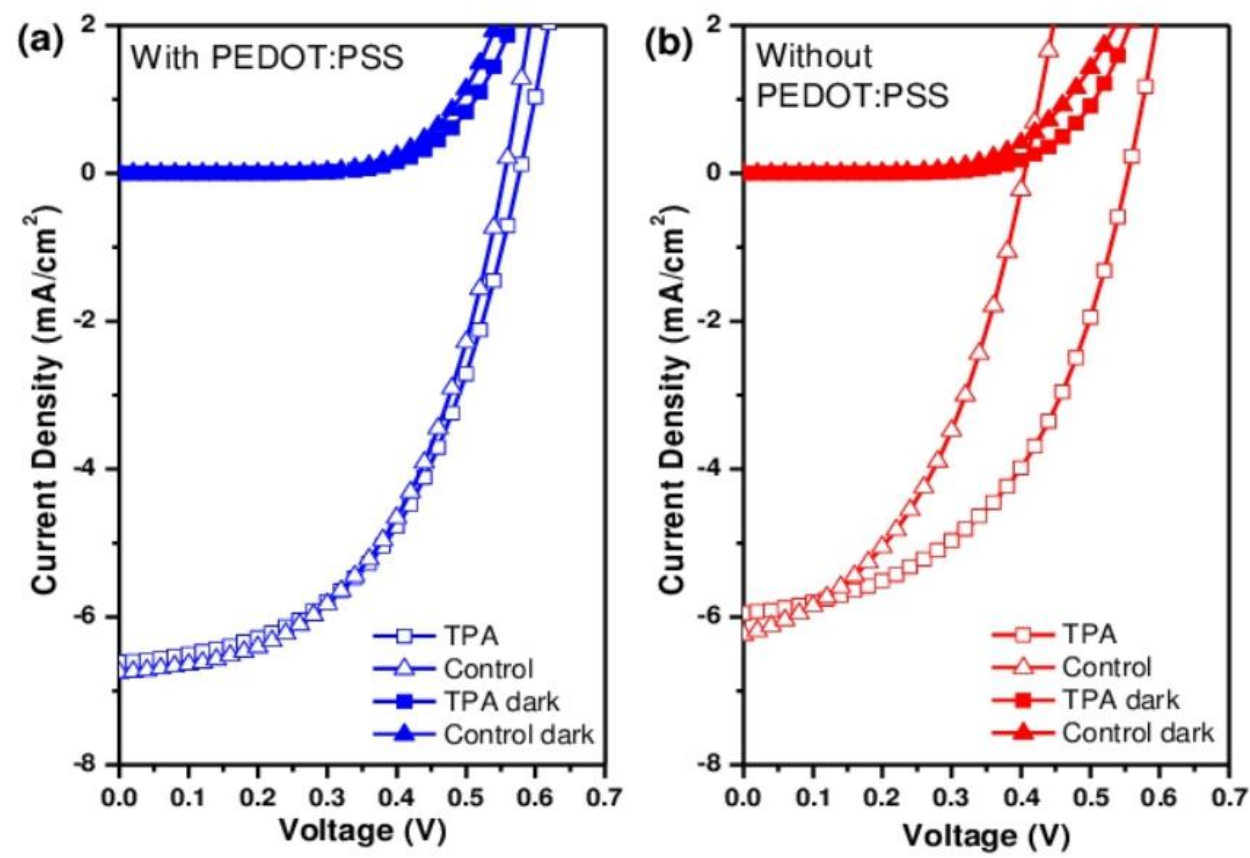

Fig. 3 Current-voltage (J-V) curves of TPA-SAM modified P P $_{3}$ HT-PCBM based annealed solar cells (a) with and (b) without PEDOT:PSS buffer layer.

Active blend of regioregular poly(3-hexylthiophene) $\left(\mathrm{P}_{3} \mathrm{HT}\right)$ (Merck, $\left.\mathrm{Mw}=26200\right)$ and $(6,6)$-phenyl $\mathrm{C}_{61}$-butyric acid methyl ester (PCBM) was prepared from their dichlorobenzene solutions with concentrations $(10 \mathrm{mg} / \mathrm{mL})$.

The solutions were spin-coated on the TPA modified and 6 patterned ITO-coated glass substrates $(500 \mathrm{rpm}, 90 \mathrm{~s})$ and $\mathrm{P}$ were transferred into glovebox with $\mathrm{Al}$ electrode thermal evaporation facility. Thermal annealing of devices $\left(140^{\circ} \mathrm{C}\right.$ for $30 \mathrm{~min}$ ) was done inside the glovebox after the deposition of the electrode. All of the devices were encapsulated with a glass cap using an ultraviolet curing epoxy resin. The $15 \mathrm{~min}$ exposure to the $\mathrm{UV}$ radiation was performed from the $\mathrm{Al}$ metal side to avoid photodegradation of the organic layers across the active region. The device structure was Glass/ITO/TPA SAM/ $\mathrm{P}_{3} \mathrm{HT}$ :PCBM/Al as shown in Fig. 2. Control devices with and without PEDOT:PSS (no TPA SAM) were also made by spincoating. The current-voltage characteristics of the encapsulated devices were measured outside the glovebox under simulated AM1.5 solar illumination at $100 \mathrm{~mW} \mathrm{~cm}^{-2}(1$ sun), using a semiconductor characterization system (SCS4200, Keithley Instruments Inc.) at room temperature.

\section{Result and Discussion}

Fig. 3(a-b) shows the photocurrent $(J)$-voltage $(V)$ curves of these devices with and without PEDOT:PSS layer as indicated. Curves for dark current are also shown for reference. Control device without PEDOT:PSS showed the $J \mathrm{sc}, V \mathrm{oc}, F F$, power conversion efficiency $(\eta)$ values as $6.20 \mathrm{~mA} \mathrm{~cm}^{-2}, 0.40 \mathrm{~V}, 0.44$ and $1.10 \%$, respectively and that with TPA showed $5.95 \mathrm{~mA}$ $\mathrm{cm}^{-2}, 0.55 \mathrm{~V}, 0.50$ and $1.61 \%$. Although there was little but not significant decrease in photo-current, the later showed considerable increase in the Voc and fill factor and power conversion efficiency $(\eta)$ was also increased from 1.10 to $1.61 \%$. The control device with PEDOT:PSS buffer layer showed comparatively better performance with $J_{\mathrm{sc}}, V_{\mathrm{oc}}, \eta$ at $6.75 \mathrm{~mA} \mathrm{~cm}{ }^{-2}, 0.56 \mathrm{~V}, 1.88 \%$, respectively. This device performance was increased when both the TPA-SAM and PEDOT:PSS were used as buffer layers in combination. The Voc was increased from 0.56 to $0.58 \mathrm{~V}$ and the PCE was also improved from 1.88 to $1.92 \%$.

Table 1. Comparative performance of various kinds of photovoltaic devices fabricated.

\begin{tabular}{ccccc}
\hline & $\mathrm{Jsc}\left(\mathrm{mA} / \mathrm{cm}^{2}\right)$ & Voc $(\mathrm{V})$ & $\mathrm{FF}$ & $\eta(\%)$ \\
\hline Control & 6.20 & 0.40 & 0.40 & 1.10 \\
TPA-SAM & 5.95 & 0.55 & 0.50 & 1.61 \\
Control- PEDOT:PSS & 6.75 & 0.56 & 0.50 & 1.88 \\
$\begin{array}{c}\text { TPA- } \\
\text { SAM:PEDOT:PSS }\end{array}$ & 6.61 & 0.58 & 0.50 & 1.92 \\
\hline
\end{tabular}

All these devices with respect to their photovoltaic parameters are summarised in Table 1. Taking into consideration all these comparative parameters we can say that device with terephthaldehyde SAM showed comprable performance over the one with PEDOT:PSS alone. This is attributed to the better wettability of TPA-SAM layer (performed scotch tape test). The work function for the bare ITO was found to be $4.85 \mathrm{eV}$ and it was increased significantly 

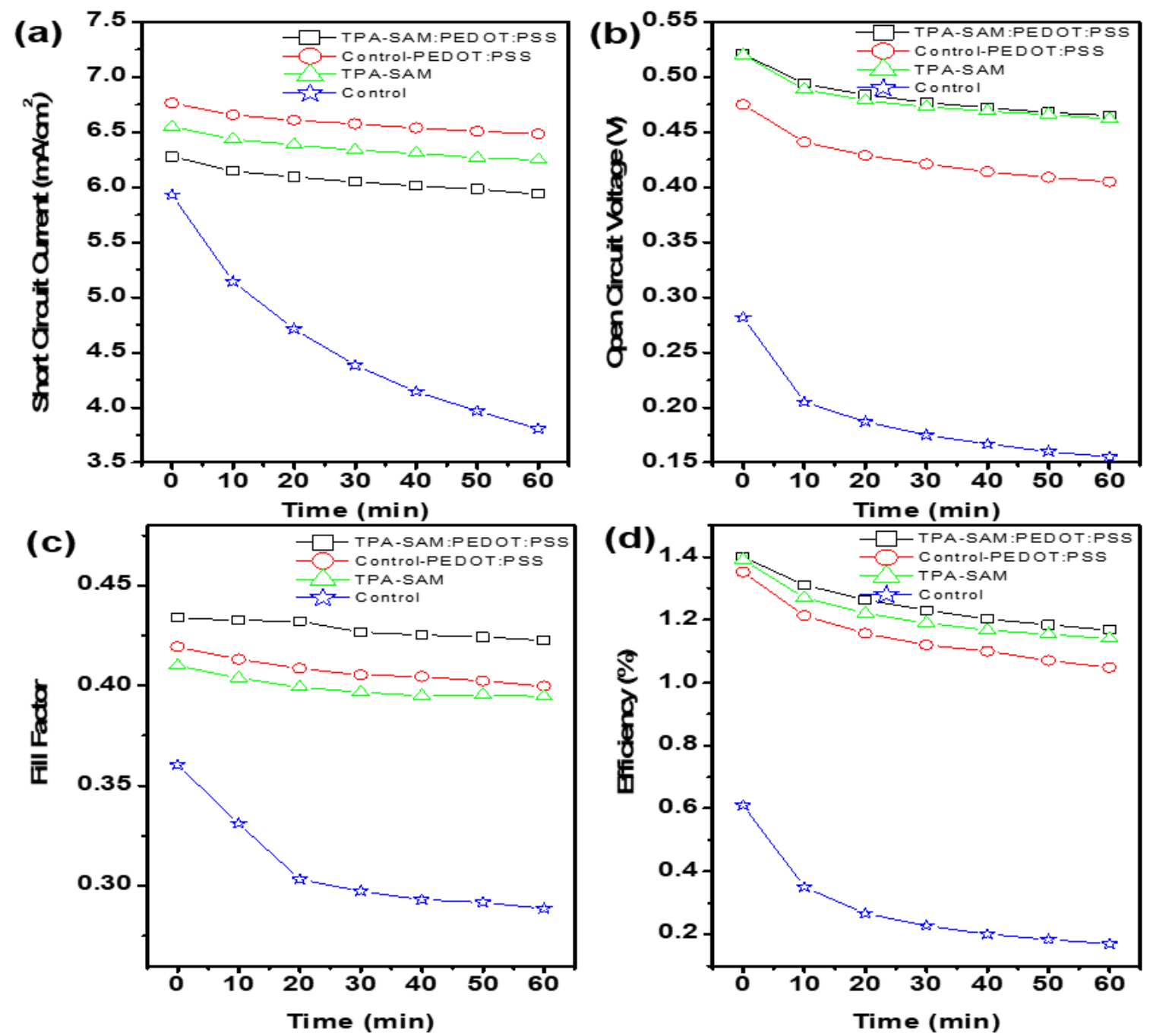

Fig. 4 Graphs of the stability studies under one sun illumination for an hour of the devices prepared with different architectures as shown.

to $5.07 \mathrm{eV}$ when modified with TPA-SAM (see Fig. S1 of Supplimentary Information) and this resulted in increase in Voc from 0.40 to $0.55 \mathrm{~V}$. The electrode work functions are determined using AC-2 (Riken Keiki). Kim et al. ${ }^{[18]}$ have shown that the modification of the ITO sustrate has two major effects on $\mathrm{P}_{3} \mathrm{HT}:$ PCBM heterojunction solar cells, i.e., changes in the hole injection barrier because of changes in the work function of ITO, and changes in the morphology of the active layer. In our devices, there is no morphology effect on $\mathrm{P}_{3} \mathrm{HT}$ :PCBM, since PEDOT:PSS layer is contacting to this layer even though TPA SAM was inserted. The self-assembled monolayers of polar molecules can be used in order to provide work functions intermediate between that of ITO and of PEDOT:PSS coated ITO electrode. ${ }^{[16]}$ Although, PEDOT: PSS is widely used as a buffer layer on top of ITO electrodes to improve the hole injection in polymer electronic devices, there are concerns about chemical reactions ${ }^{[16]}$ possibly occurring at the PEDOT: PSS interface and their effects on device lifetime. PEDOT:PSS is hydrophilic and its aqueous dispersions are at $\mathrm{pH} \sim 1$ and are corrosive to ITO anode that would degrade long- term stability of the polymer PV cells ${ }^{[19]}$ and it is evident from the stability studies we carried out after six months of device fabrication under continuous illumination of white light for an hour on these devices (Fig. 4), which shows deterioration of the devices fabricated without TPA-SAM and PEDOT:PSS as buffer layer. Although all the devices were degraded under storage over the period, the stability of the device with just TPA-SAM was better compared to other devices as it showed lesser decrease in the efficiency viz., from 1.61 to $1.40 \%$ and this indicates the role of PEDOT:PSS in aiding the deterioration. Devices with TPA-SAM showed better performance and stability over the ones without TPA-SAM with respect to Voc and efficiency $(\eta)$, as shown in Fig. 4-b and $\mathrm{d}$ above. Thus it is clear that, we can prevent the reaction between ITO and PEDOT:PSS by effectively inserting TPASAM in between by acetalization and that increases the lifetime of the device.

\section{Conclusion}

In conclusion, an acetalization reaction was used as a versatile 
method to immobilize terephthaldehyde molecules on the ITO surface to form the TPA-SAM which provides the possibility of fine tuning the barrier height for hole injection in a relatively straight forward way and ultimately the means to fabricate all-organic solution processed solar cells. The TPASAM-PEDOT:PSS device fabricated under unoptimised conditions showed power conversion efficiency $(\eta)$ of $1.92 \%$ and better stability. The formation of high-quality SAM using acetalization reaction opens up way to control electronic states of electrode/organic interafces aiding to better device performance with further optimization.

\section{Acknowledgement}

C.J.B. would like to thank Science and Engineering Research Board (SERB), DST, India for support under the research grant SB/FT/CS-056/2013 and acknowledges Japan Society for Promotion of Science (JSPS) for support.

\section{Supporting information}

Applicable, Electronic Supplementary Information (ESI) available: Graphs of the work-function values obtained for clean ITO surface and TPA-SAM modified ITO surface.

\section{Conflict of interest}

There are no conflicts to declare.

\section{References}

[1] (a) K. Kim, J. Liu, M. Namboothiry, D. Carroll, Appl. Phys. Lett., 2007, 90, 163511, doi: 10.1063/1.2730756 (b) B. C. Thompson, J. M. J. Frechet, Angew. Chem. Int. Ed., 2008, 47, 5877, doi: 10.1002/anie.200702506.

[2] Y. Yan, X. Liu, T. Wang, Adv. Mater., 2017, 29, 1-22, doi: 10.1002/adma.201601674.

[3] (a) Sheng Bi, Zhongliang Ouyang, Shoi.eb Shaik, D. Li, Sci Rep., 2018, 8, 9574, doi: 10.1038/s41598-018-27868-2. (b) S. Bi, Z. Ouyang, Q. Guo, C. Jiang, RSC Adv., 2018, 8, 6519-6526, doi: 10.1039/C7RA13219K.

[4] P. Schilinsky, C. Waldauf, C. Brabec, Appl. Phys. Lett., 2002, 81, 3885-3887, doi: 10.1063/1.1521244.

[5] F. Padinger, R. S. Rittberger, N. S. Sariciftci, Adv. Funct. Mat., 2003, 13, 85-88, doi: 10.1002/adfm.200390011.

[6] (a) W. Ma, C. Yang, X. Gong, K. Lee, A. J. Heeger, Adv. Funct. Mat., 2005, 15, 1617-1622, doi: 10.1002/adfm.200500211. (b) G. Li, V. Shrotyiya, J. Huang, Y. Yao, T. Moriarty, K. Emery, Y. Yang, Nat. Mater., 2005, 4, 864868, doi: 10.1142/9789814317665_0012.

[7] Bright Walker, Arnold B. Tamayo, Xuan-Dung Dang, Peter Zalar, Jung Hwa Seo, Andres Garcia, Mananya Tantiwiwat, T.-Q. Nguyen, Adv. Funct. Mater., 2009, 19, 3063-3069, doi: 10.1002/adfm.200900832.

[8] Q. Jiang, T. Xu, Comment. Inorg. Chem., 2016, 36, 200-214, doi: 10.1080/02603594.2015.1116985.
[9] (a) R. Lohwasser, J. Bandara, M. Thelakkat, J. Mater. Chem., 2009, 19, 4126, doi: 10.1039/B900921C. (b) J. Jo, S.-I. Na, S.-S. Kim, T.-W. Lee, Y. Chung, S.-J. Kang, D. Vak, D.-Y. Kim, $A d v$. Funct. Mat., 2009, 19, 2398, doi: 10.1002/adfm.200900183. (c) Y.-T. Chang, S.-L. Hsu, M.-H. Su, T. A. Singh, E. W. G. Diau, K.H. Wei, Adv. Funct. Mat., 2008, 18, 2356, doi: 10.1002/adfm.200701150.

[10] J. S. Kim, M. Granstrom, R. H. Friend, N. Johansson, W. R. Salaneck, R. Dia, W. J. Feast, F. Cacialli, J. Appl. Phys., 1998, 84, 6859, doi: 10.1063/1.124403.

[11] S. Khodabakhsh, B. M. Sanderson, J. Nelson, T. S. Jones, Adv. Funct. Mat., 2006, 16, 95-100, doi: 10.1002/adfm.200500207.

[12] A. C. Arias, L. S. Roman, T. Kugler, R. Toniolo, M. S. Meruvia, I. A. Hummelgen, Thin Solid Film, 2000, 371, 201, doi: 10.1016/S0379-6779(98)01127-8.

[13] J. S. Kim, F. Cacialli, M. Granstrom, R. H. Friend, N. Johansson, W. R. Salaneck, R. Daik, W. J. Feast, Synth. Met., 1999, 101, 111, doi: 10.1016/S0040-6090(00)00967-6.

[14] T. M. Brown, J. S. Kim, R. H. Friend, F. Cacialli, R. Daik, W. J. Feast, Synth. Met., 2000, 111, 285, doi: org/10.1063/1.1394920.

[15] S. F. J. Appleyard, S. R. Day, R. D. Pickford, M. R. Willis, J. Mater. Chem., 2000, 10, 169, doi: 10.1039/A903708J.

[16] S. Khodabakhsh, D. Poplavskyy, S. Heutz, J. Nelson, D. D. C. Bradley, H. Murata, T. S. Jones, Adv. Funct. Mat., 2004, 14, 1205-1210, doi: 10.1002/adfm.200400035.

[17] T. Nakayama, T. Matsushima, H. Murata, Thin Solid Films, 2009, 518, 739-742, doi: 10.1016/j.tsf.2009.07.081.

[18] J. S. Kim, J. H. Park, J. H. Lee, J. Jo, D.-Y. Kim, K. Cho, Appl. Phys. Lett., 2007, 91, 112111, doi: 10.1063/1.2778548.

[19] K. W. Wong, H. L. Yip, Y. Luo, K. Y. Wong, W. M. Lau, K. H. Low, H. F. Chow, Z. Q. Gao, W. L. Yeung, C. C. Chang, Appl. Phys. Lett., 2002, 80, 2788-2790, doi: 10.1063/1.1469220.

\section{Author Information}

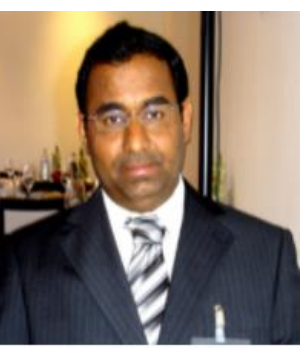

Dr. Chetan Bhongale is currently an Assistant Professor at the Defence Institute for Advanced Technology (DU), DIAT, Pune, India. Prior to that, he was a faculty in University of Pune. Dr. Bhongale's previous professional affiliations include post-doctoral researcher at UNLV, Las Vegas, USA, University of Bayreuth, Germany and Japan Advanced Institute of Science and Technology, Japan. 


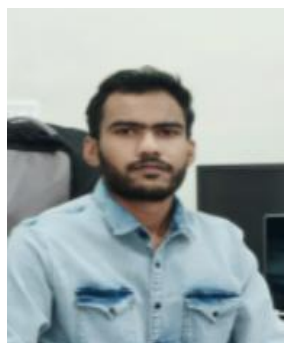

Rahul Chaudhari is a research scholar at Department of Applied Chemistry of Defence Institute of Advanced Technology (DIAT), Pune, India. His work focuses on organic-inorganic hybrid mesoscopic materials. He holds post-graduate degree in polymer chemistry from KBCNMU, Jalgaon, India.

Publisher's Note: Engineered Science Publisher remains neutral with regard to jurisdictional claims in published maps and institutional affiliations. 